\title{
Static and Dynamic of Rotating Bose-Einstein Condensates in Synthetic Magnetic Field
}

\author{
Yu Lan, Jingming Hua, Bing Wang, Genwang Fan and Qiang ZhaO* \\ Department of Applied Physics, North China University of Science and Technology, Tangshan 063210, China \\ (Received November 23, 2017; in final form May 22, 2018)

\begin{abstract}
We consider theoretically static and dynamic properties of vortex formation in trapped Bose-Einstein condensates in synthetic magnetic field and compare the results created by rotating frame method. As the first step, we discuss the ground state properties and show that the number of vortices and rotational frequency is a linear relation. We further deduce the analytical results and find it qualitatively agrees with numerical solutions. In addition, we explore the vortex dynamics, focusing on the difference between the two rotational methods. Numerical results indicate that synthetic magnetic field is more difficult to add a large angular momentum. However, this issue can be effectively improved by increasing the contact interaction.
\end{abstract}

DOI: 10.12693/APhysPolA.134.503

PACS/topics: 67.85.-d, 03.75.Kk, 03.75.Lm

\section{Introduction}

Quantized vortices in the atomic Bose-Einstein condensates (BECs) has attracted much research interest [15]. The atomic BECs are good prototypes for investigations of phenomena relevant to quantized vortices because of the following properties. Firstly, BECs provide an ideal impurity-free environment for the research of physicists. Secondly, weak interaction between the condensate atoms makes theoretical treatments easier. This system is well described by the Gross-Pitaevskii (GP) equation [6]. Thirdly, many physical parameters of BECs are experimentally controllable. Therefore, it is possible to visualize directly the BEC clouds and quantized vortices $[7,8]$.

BECs respond to rotation by creating quantized vortices. Conventionality, vortices can be created by means of rotating trap, i.e. rotating frame $[7,8]$. When the trapped condensate is driven to rotate, singly quantized vortices form. The number of vortices entering the condensate above a certain critical velocity of rotation increases with growing rotational frequency, until a stationary Abrikosov lattice is formed. Some experimental groups reported vortex lattice structures under the rotating potential $[9,10]$. In addition, much theoretical work has confirmed by the numerical simulation of GP equation that the ground state of BECs under rotation is a regular Abrikosov triangular lattice [11-19], and such lattices contain hundreds of vortices.

Recently, the synthetic magnetic field technique appears as another outstanding tool, which enables us to form the vortex lattice in a BECs [20-22]. When the synthetic magnetic field exceeds the critical value, quantum vortices are created. The novel technique opens the possibility for more careful investigation of the new method

*corresponding author; e-mail: zhaoqiangac2004@sina.com of the vortex formation. Moreover, the author argued that such approach could effectively add large angular momentum compared with the conventional method, and it can overcome the heating and metastability of the condensate.

The main motivation of the present manuscript is to study how the vortex formation is affected in synthetic magnetic field by the free parameters, such as rotational frequency and atom interactions. We compare our results with the rotating frame case. To our knowledge, the number of vortices $N_{v}$ and the rotational frequency $\Omega$ appear to be well described by a quadratic relation in rotating frame. However, in synthetic magnetic field, a linear behavior is observed, and no attempt has been made so far to explore the analysis relation. In this paper, we give a expression about $N_{v} \sim \Omega$, which can explain this point qualitatively. In addition, the dependence on the approximate time for the formation of steady state of vortices, angular momentum and the number of vortices on the inter-atomic interaction have been studied. We find that the rotational efficiency can be effectively enhanced at sufficiently large contact interaction.

The rest of the paper is structured as follows. In Sect. 2, we outline the derivation of the effective 2D model under synthetic magnetic field, starting from the 3D GP equation. In Sect. 3, numerical solutions for number of vortices in two different scenarios, synthetic magnetic field and rotating frame, are produced. Next, an analytical solution is obtained from the Feynman rule. The analytical solution makes it also possible to predict the relation of the number of vortices and rotational frequency, which is an essential result. Further, in Sect. 4, we display vortex dynamics, the effect of inter-atomic interaction on the dynamics behavior is discussed. The paper is concluded by Sect. 5 .

\section{Theoretical model}

At zero temperature, the static and dynamic properties in a synthetic magnetic field can be well described 
by the mean-field GP equation

$$
\begin{aligned}
& \mathrm{i} \hbar \frac{\partial \psi(\boldsymbol{r}, t)}{\partial t}= \\
& \quad\left[\frac{1}{2 m}(\boldsymbol{P}-\boldsymbol{A})^{2}+V(\boldsymbol{r})+g|\psi|^{2}\right] \psi(\boldsymbol{r}, t) .
\end{aligned}
$$

Here $\boldsymbol{P}$ is the canonical momentum operator, $\boldsymbol{A}$ is the synthetic gauge potential. We assume that the synthetic field $\boldsymbol{B}=2 m \Omega e_{z}$ is parallel to the $z$ axis and choose the symmetric gauge $\boldsymbol{A}=(\boldsymbol{B} \times \boldsymbol{r}) / 2=m\left(\omega_{l} \times \boldsymbol{r}\right)$, where $\omega_{l}$ is the Larmor frequency. $\psi(\boldsymbol{r}, t)$ is the condensate wave function, $m$ is the atomic mass, and $V(\boldsymbol{r})=$ $\frac{1}{2} m \omega_{\perp}^{2}\left(x^{2}+y^{2}\right)+\frac{1}{2} m \omega_{z}^{2} z^{2}$ is the confining potential. The contact interaction is represented by $g=4 \pi \hbar^{2} a_{s} / m$, where $a_{s}$ is the $s$-wave scattering length. The norm of the wave function is fixed by total number of atoms $N=\int \mathrm{d}^{3} \boldsymbol{r}|\psi(\boldsymbol{r}, t)|^{2}$.

The 3D GP equation can be reduced into an effective $2 \mathrm{D}$ equation, provided that the confinement in the $z$-direction is strong enough. To this end, we assume that the $3 \mathrm{D}$ wave function is factorized as $\psi(\boldsymbol{r}, t)=\psi(x, y, t) \phi(z) \exp (-\mathrm{i} \mu t / \hbar) . \quad \phi(z)=$ $\left(\pi l^{2}\right)^{-1 / 4} \exp \left(-z^{2} / 2 l^{2}\right)$ is the ground state of harmonic oscillator along the $z$-direction with the characteristic length $l=\sqrt{\hbar / m \omega_{\perp}}$. In this work, length, time, energy, angular momentum, and rotation angular frequency are in units of $l, 1 / \omega_{\perp}, \hbar \omega_{\perp}, \hbar$, and $\omega_{\perp}$, respectively. Then the effective $2 \mathrm{D}$ equation can be obtained after integrating over the $z$-coordinate

$$
\text { i } \frac{\partial \psi}{\partial t}=\left[\frac{1}{2}(\boldsymbol{P}-\boldsymbol{A})^{2}+\frac{1}{2}\left(x^{2}+y^{2}\right)+g_{x y}|\psi|^{2}\right] \psi,
$$

where $\beta=4 \pi N a_{s} / l, g_{x y}=\beta \sqrt{\gamma_{z} / 2 \pi}$ represents the effective 2D interaction strength. $\gamma_{z}=\omega_{z} / \omega_{\perp}$ is the trap aspect ratio of the anisotropic trap.

Similarly, the corresponding 2D dimensionless GP equation for BECs in rotating frame can be written as

$$
\mathrm{i} \frac{\partial \psi}{\partial t}=
$$

$\left[\frac{1}{2}(\boldsymbol{P}-\boldsymbol{A})^{2}+\frac{1}{2}\left(1-\Omega^{2} / \omega_{\perp}^{2}\right)\left(x^{2}+y^{2}\right)+g_{x y}|\psi|^{2}\right] \psi$,

where $\Omega$ is the rotational frequency.

Equations (2) and (3) can be solved numerically by norm-preserving imaginary time propagation method on a $256 \times 256$ square grid [23]. The propagation continues until the fluctuation in the norm of the wave function becomes smaller than $10^{-6}$. The spatial- and time-step sizes employed are 0.05 and 0.001 , respectively. We prepare the initial wave function as a combination of the ground state of harmonic potential and a vortex state, which can be written as

$$
\phi(x, y, 0)=\frac{(1-\Omega) \phi_{h o}(x, y)+\Omega \phi_{h o}^{v}(x, y)}{\left\|(1-\Omega) \phi_{h o}(x, y)+\Omega \phi_{h o}^{v}(x, y)\right\|},
$$

with

$$
\psi_{h o}(x, y)=\frac{1}{\sqrt{\pi}} \mathrm{e}^{-\left(x^{2}+y^{2}\right) / 2},
$$

$$
\psi_{h o}^{v}(x, y)=\frac{x+\mathrm{i} y}{\sqrt{\pi}} \mathrm{e}^{-\left(x^{2}+y^{2}\right) / 2} .
$$

In the numerical simulations, our results show that such initial data always gives the minimum energy of the system.

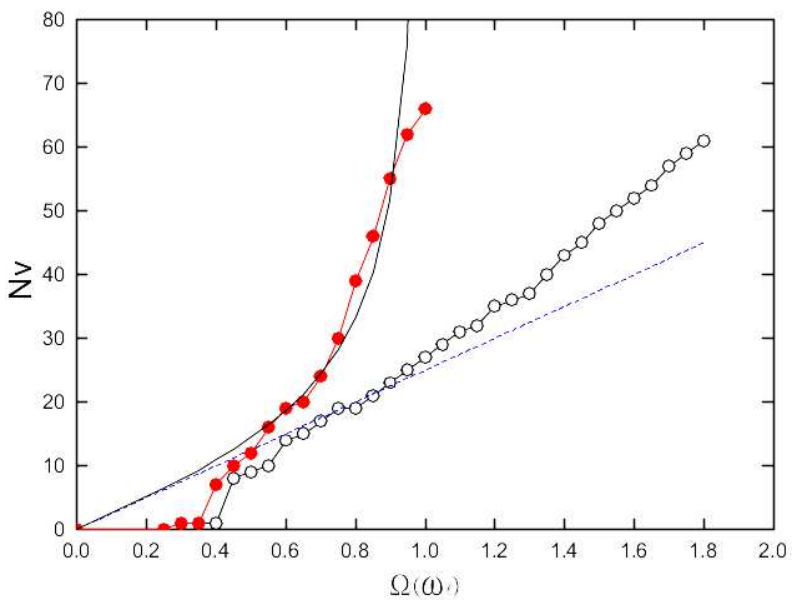

Fig. 1. Equilibrium number of vortices $N_{v}$ as a function of rotational frequency (the Larmor frequency) $\Omega\left(\omega_{l}\right)$ at $g_{x y}=1000$. The empty circle points and filled circle points represent the numerical results for synthetic magnetic field and rotating frame from Eqs. (2) and (3), respectively. The dashed and solid curves correspond to Eqs. (9) and (10), respectively.

\section{Ground state}

It has been known that the relation between number of vortices and rotational frequency is the critically important issue in rotating quantum gas. To simplify the numerical simulations, and highlight the effects of the rotational frequency, we fix the contact interaction $g_{x y}=1000$.

Figure 1 shows the typical example of the dependence of number of vortices $N_{v}$ on the rotational frequency (the Larmor frequency) $\Omega\left(\omega_{l}\right)$ at $g_{x y}=1000$. The empty circle points and filled circle points represent the numerical results for synthetic magnetic field and rotating frame, respectively. We find two different development of the number of vortices. For rotating frame, we observe that $N_{v}$ increases quadratically with $\Omega$ and diverges at $\Omega=\omega_{\perp}$. For synthetic magnetic field, the number of vortices increases linearly with increase of $\omega_{l}$.

To present a clearer illustration on these trends, we explore the analytical analysis. For a superfluid rotating in a rigid container with radius $R$, the number of vortices $N_{v}$ can be determined by the Feynman rule $N_{v}=m \Omega R^{2}(\Omega) / \hbar[24]$. Also, when the interaction energy is large compared to the kinetic energy, the suitable analytical treatment of the present problem is that the wave function may be described by the Thomas-Fermi approximate. We solve the time-independent GP Eq. (3) for the condensate wave function 


$$
|\psi(\boldsymbol{r})|^{2}=\frac{1}{g_{x y}}\left(\mu-\frac{1}{2} m\left(\omega_{\perp}^{2}-\Omega^{2}+\omega_{l}^{2}\right) r^{2}\right) .
$$

Then, the normalization condition determines the chemical potential as

$$
\mu(\Omega)=\mu(0) \sqrt{1-\Omega^{2} / \omega_{\perp}^{2}+\omega_{l}^{2} / \omega_{\perp}^{2}},
$$

with $\mu(0)=N g_{x y} m \omega_{\perp}^{2} / \pi$. In the spirit of the ThomasFermi approximate, the radius

$$
R^{2}(\Omega)=\frac{R^{2}(0)}{\sqrt{1-\Omega^{2} / \omega_{\perp}^{2}+\omega_{l}^{2} / \omega_{\perp}^{2}}},
$$

where $R^{2}(0)=\sqrt{4 N g_{x y} / \pi m \omega_{\perp}^{2}}$. Since the Larmor frequency has the same physical meaning with rotational frequency in both rotation, it is sufficient to consider $\omega_{l}=\Omega$. Therefore, quite naturally, the chemical potential and radius may be written as $\mu(\Omega)=\mu(0)$ and $R(\Omega)=R(0)$. The number of vortices is given by

$$
N_{v}=\frac{m \Omega}{\hbar} R^{2}(0) \text {. }
$$

From (9), we can see the intuitive understanding on the relation of the rotational frequency to number of vortices. $N_{v}$ increases linearly with increasing $\Omega$, i.e., $N_{v} \propto \Omega$.

Similarly, in rotating frame case, $\mu(\Omega)=$ $\mu(0) \sqrt{1-\Omega^{2} / \omega_{\perp}^{2}}$ and $R^{2}(\Omega)=\frac{R^{2}(0)}{\sqrt{1-\Omega^{2} / \omega_{\perp}^{2}}}$, the number of vortices is calculated

$$
N_{v}=\frac{m \Omega}{\hbar} R \frac{R^{2}(0)}{\sqrt{1-\Omega / \omega_{\perp}}} .
$$

In this paper, we focus on the scaling property of $N_{v}$ with respect to $\Omega$. Before the further analytical analysis, we set the fitting parameter $R(0)=5.0$. In fact, it is a somewhat arbitrary value for a trapped BECs. We plot the number of vortices taken from analytical analysis in Fig. 1. The dashed and solid curves correspond to Eqs. (9) and (10), respectively. We find that the analytical results match well with numerical simulations. For synthetic magnetic field, when $\Omega>1.2 \omega_{\perp}$, the analytical results are about $20 \%$ lower than the numerically obtained results. This is probably due to the inhomogeneous density [6].

Finally, we also notice that the chemical potential is irrespective of the value of rotational frequency in synthetic magnetic field, and it decreases continuously in rotating frame. As expected, such properties have been clearly depicted by the numerical calculation in Ref. [25].

\section{Dynamics}

Formation of vortices process of a condensate exhibits interesting dynamics. The synthetic magnetic field is also fascinating system to study such dynamics because the process is much different from the rotating frame case. Here we focus on the difference between the two rotational methods.

We initially prepare the stable ground state wave function for $\Omega=0$. The vortex formation is then studied by replacing the term $\mathrm{i} \partial / \partial t$ with $(\mathrm{i}-\gamma) \partial / \partial t$ in in the left-hand side of Eqs. (2) and (3) and introducing of $\Omega$. This form of the dissipative equation follows the study of Choi et al. [26]. Choi et al. determined the value of $\gamma$ by fitting their theoretical results with the MIT experiments [27]. Thus, we introduce the dissipation in the dynamics discussion. The phenomenological dissipative may be related to the coupled dynamics of a condensate and a noncondensate, i.e. zero temperature and non-zero temperature. Since this dissipative term is much smaller than other terms in the GP equation, a small variation of $\gamma$ does not change the dynamics qualitatively but only modifies the relaxation time scale. In addition, our results show that when $\gamma$ is set to zero the vortices in condensate do not exist.

In Fig. 2 we show the snapshots of the density contours and phase patterns for $\Omega=0.7 \omega_{\perp}$ and $\gamma=0.03$. For rotating BECs, when it begins to rotate the surface becomes unstable and ripples are developed on the surface as time progress. Then these ripples gradually improve into vortices and reach a stable configuration at a finite time. Here we compare the time taken for the formation of steady state vortices for both rotation. Figures $2 \mathrm{a}-\mathrm{c}$ and $\mathrm{d}-\mathrm{f}$ show the development of vortices for $g_{x y}=1000$ and $g_{x y}=2000$ in rotating frame, respectively. The steady state phase pattern of final wave function is shown in the fourth columns. For weak contact interaction, such as $g_{x y}=1000$, the surface ripples are formed at $t \approx 90 / \omega_{\perp}$ and an equilibrium state of 20 vortices are formed. For a larger $g_{x y}=2000$, the surface ripples are formed more rapidly, the time $t \sim 60 / \omega_{\perp}$ and a very stable pattern with 31 vortices are created.

Figures $2 \mathrm{~h}-\mathrm{j}$ and $\mathrm{k}-\mathrm{m}$ show the development of vortices for synthetic magnetic field case at $g_{x y}=1000$ and $g_{x y}=$ 2000 , respectively. For $g_{x y}=1000$, the surface ripples are formed at time $t \approx 220 / \omega_{\perp}$, which is much slower when compared to that of rotating frame case discussed above. Finally, an equilibrium state of 12 vortices are formed. On the other hand, the surface ripples are observed at $t \approx 100 / \omega_{\perp}$ when applying $g_{x y}=2000$, and a very stable pattern with 17 vortices is created.

To gain a closer insight into vortex formation process, in Fig. 3 we plot the time evolution of angular momentum $L_{z}$ per atom as a function of time, which is defined as:

$$
L_{z}=\mathrm{i} \iint \psi^{\star}\left(y \partial_{x}-x \partial_{y}\right) \psi \mathrm{d} x \mathrm{~d} y \text {. }
$$

The blue and red represent the numerical results for synthetic magnetic field and rotating frame, respectively. The dashed and solid curves correspond to the contact interaction 1000 and 2000, respectively. We find that the angular momentum $L_{z}$ per atom gradually increases and then settles to a steady value. The increase in the $L_{z}$ is associated with the entry of vortices into the condensate. When the system reaches steady state, it is seen that the value of $L_{z}$ in rotating frame is about twice as large as that in synthetic magnetic field case. Also, the time taken is smaller in rotating frame case. As a comparison, we also show the time evolution of $L_{z}$ at $\gamma=0.08$. The symbol $\gamma_{r}$ and $\gamma_{s}$ represent the dissipation in rotating frame and synthetic magnetic field, respectively. Figures $4 \mathrm{a}-\mathrm{e}$ correspond to $g_{x y}=1000,2000,3000,4000$, and 5000 , 


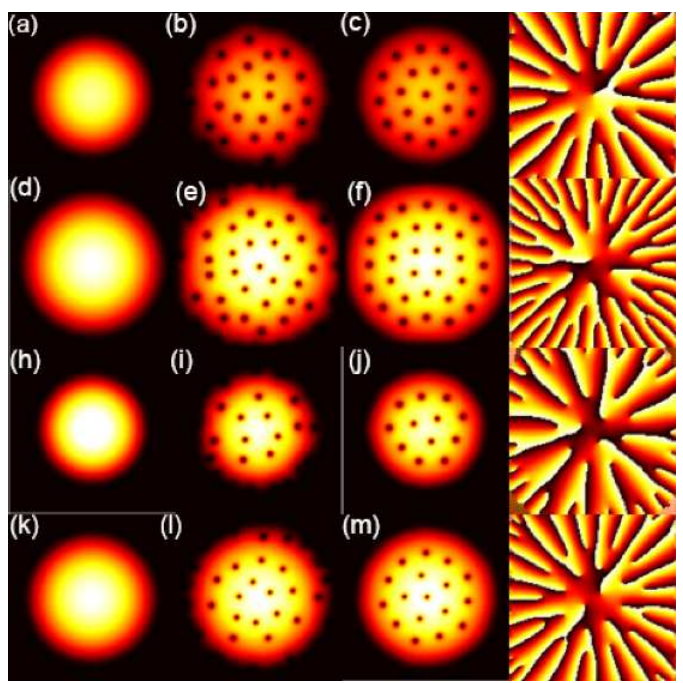

Fig. 2. Contour plots of the densities, showing the development of vortices in rotating frame case, for $g_{x y}=$ 1000: (a) $t=0$, (b) $t=130 / \omega_{\perp}$, (c) $t=300 / \omega_{\perp}$, for $g_{x y}=2000$, (d) $t=0$, (e) $t=70 / \omega_{\perp}$, (f) $t=300 / \omega_{\perp}$, synthetic magnetic field case for $g_{x y}=1000$, (h) $t=0$, (i) $t=250 / \omega_{\perp}$, (j) $t=300 / \omega_{\perp}$, for $g_{x y}=2000,(\mathrm{k})$ $t=0$, (l) $t=120 / \omega_{\perp},(\mathrm{m}) t=300 / \omega_{\perp}$. Note that the fourth columns are the phase distributions of steady state. The rotational frequency is fixed at $\Omega=0.7 \omega_{\perp}$.

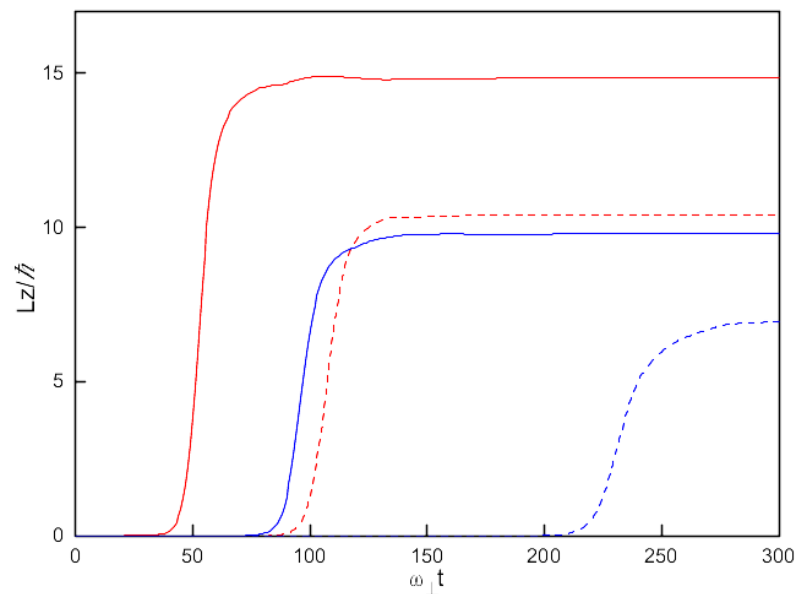

Fig. 3. Time evolution of the angular momentum per atom, during the development of vortices shown in Fig. 2. The blue and red represent the numerical results for synthetic magnetic field and rotating frame, respectively. The dashed and solid curves correspond to the strength of the contact interaction 1000 and 2000, respectively.

respectively. It is obvious that the large dissipation only change the relaxation process quantitatively. The value of $L_{z}$ remain almost unchanged. Moreover, by simply counting, we also verify that the number of vortices $N_{v}$ is the same as that of $\gamma=0.03$.

The above observation clearly indicates that the synthetic magnetic field rotates the condensate less effi-
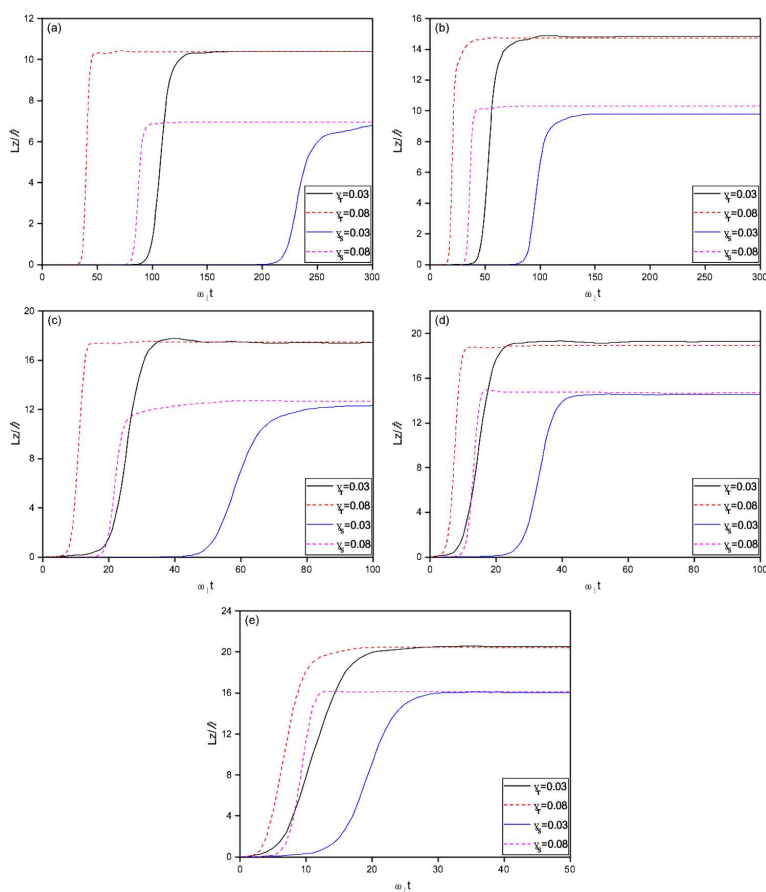

Fig. 4. The time development of angular momentum per atom with dissipation parameter 0.03 and 0.08 . The symbol $\gamma_{r}$ and $\gamma_{s}$ represents the dissipation in rotating frame and synthetic magnetic field, respectively. (a), (b), (c), (d), and (e) correspond to $g_{x y}=1000,2000$, 3000,4000 , and 5000, respectively.

ciently. To overcome this difficulty, we further increase the contact interaction to sufficiently large value. We estimate the approximate time $t_{v o r}$ for the formation of steady state of vortices with the rotational frequency $\Omega=0.7 \omega_{\perp}$. Table I and Table II show $t_{\text {vor } 1}(\gamma=0.03)$ and $t_{\text {vor } 2}(\gamma=0.08)$, angular momentum per atom $L_{z}$ and the number of vortices $N_{v}$ in synthetic magnetic field and rotating frame for the creation of equilibrium vortices at different contact interactions $g_{x y}$, respectively. We find that the time taken for the creation of steady state vortices in synthetic magnetic field is much bigger than that in rotating frame at lower contact interaction. However, $t_{\text {vor } 1}$ and $t_{\text {vor } 2}$ is nearly equal to the same value at higher contact interaction. The ratios of the numbers of vortices in the two cases are $12 / 20,17 / 31,23 / 35$, $29 / 38,32 / 40$ and the ratios of the angular momentum per atom in the two cases are $6.946 / 10.406,9.971 / 14.839$, $12.366 / 17.431,14.558 / 19.289,16.063 / 20.647$, these ratios correspond to $g_{x y}=1000,2000,3000,4000$, and 5000 , respectively.In is clear that the number of vortices and the angular momentum per atom increases with increase of interaction, and the ratio tends to unity. These results suggest that although it is still relatively difficult to generate vortices in synthetic magnetic field compared to the rotating frame, the difference between the two cases becomes small at sufficiently large contact interactions. Actually, the $s$-wave scattering length can 
TABLE I

Values of the approximate time $t_{\text {vor } 1}(\gamma=0.03)$ and $t_{\text {vor } 2}(\gamma=0.08)$, angular momentum per atom $L_{z}$ and the number of vortices $N_{v}$ in synthetic magnetic field for the creation of equilibrium vortices at different contact interactions $g_{x y}$. Simulation parameter: $\Omega=0.7 \omega_{\perp}$.

\begin{tabular}{c|c|c|c|c}
\hline \hline$g_{x y}$ & $t_{\text {vor } 1}$ & $t_{\text {vor } 2}$ & $L_{z}$ & $N_{v}$ \\
\hline 1000 & $250 / \omega_{\perp}$ & $127 / \omega_{\perp}$ & $6.946 \hbar$ & 12 \\
2000 & $120 / \omega_{\perp}$ & $90 / \omega_{\perp}$ & $9.791 \hbar$ & 17 \\
3000 & $90 / \omega_{\perp}$ & $58 / \omega_{\perp}$ & $12.366 \hbar$ & 23 \\
4000 & $50 / \omega_{\perp}$ & $23 / \omega_{\perp}$ & $14.558 \hbar$ & 29 \\
5000 & $25 / \omega_{\perp}$ & $16 / \omega_{\perp}$ & $16.063 \hbar$ & 32
\end{tabular}

TABLE II

The same as in Table I but for rotating frame.

\begin{tabular}{c|c|c|c|c}
\hline \hline$g_{x y}$ & $t_{\text {vor } 1}$ & $t_{\text {vor } 2}$ & $L_{z}$ & $N_{v}$ \\
\hline 1000 & $130 / \omega_{\perp}$ & $64 / \omega_{\perp}$ & $10.406 \hbar$ & 20 \\
2000 & $70 / \omega_{\perp}$ & $41 / \omega_{\perp}$ & $14.839 \hbar$ & 31 \\
3000 & $50 / \omega_{\perp}$ & $30 / \omega_{\perp}$ & $17.431 \hbar$ & 35 \\
4000 & $25 / \omega_{\perp}$ & $20 / \omega_{\perp}$ & $19.286 \hbar$ & 38 \\
5000 & $20 / \omega_{\perp}$ & $14 / \omega_{\perp}$ & $20.647 \hbar$ & 40
\end{tabular}

be tuned by the Feshbach resonance technique [28, 29]. Therefore, increasing the contact interaction is an effective and realizable route to adding more angular momentum and generating more vortices in condensates rotated by a synthetic magnetic field.

\section{Conclusion}

We have studied the formation of vortices in rotating Bose-Einstein condensates in synthetic magnetic field by numerically solving the time-dependent GP equation in two dimensions. Particularly, we have calculated the number of vortices as a function of rotational frequency. It has been shown that linear behavior of a number of vortices is valid for synthetic magnetic case. Further, we have explored the influence of contact interaction on the number of vortices, angular momentum per atom, and time taken for the creation of vortices. The number of vortices and angular momentum per atom have been found to increase with an increase in the contact interaction, and the ratios in both case tend to unit. The time taken for the nucleation of vortices is estimated that strong contact interactions can nucleate the vortices very rapidly. These results imply that the rotational efficiency can be improved by increasing the strength of contact interaction.

\section{Acknowledgments}

This work is supported by the Innovation Fund (Grant No. X2017287) and Ph.D. Start-up Fund (Grant No. BS2017096) of North China University of Science and Technology.

\section{References}

[1] M.H. Anderson, J.R. Ensher, M.R. Matthews, C.E. Wieman, E.A. Cornell, Science 269, 198 (1995).

[2] K.B. Davis, M.-O. Mewes, M.R. Andrews, N.J. van Druten, D.S. Durfee, D.M. Kurn, W. Ketterle, Phys. Rev. Lett. 75, 3969 (1995).
[3] C.C. Bradley, C.A. Sackett, J.J. Tollett, R.G. Hulet, Phys. Rev. Lett. 75, 1687 (1995).

[4] N.R. Cooper, Adv. Phys. 57, 539 (2008).

[5] A.L. Fetter, Rev. Mod. Phys. 81, 647 (2009).

[6] M. Tsubota, K. Kasamatsu, M. Ueda, Phys. Rev. A 65, 023603 (2002).

[7] M.R. Matthews, B.P. Anderson, P.C. Haljan, D.S. Hall, C.E. Wieman, E.A. Cornell, Phys. Rev. Lett. 83, 2498 (1999).

[8] K.W. Madison, F. Chevy, W. Wohlleben, J. Dalibard, Phys. Rev. Lett. 84, 806 (2000).

[9] P.C. Haljan, I. Coddington, P. Engels, E.A. Cornell, Phys. Rev. Lett. 87, 210403 (2001).

[10] J.R. Abo-Shaeer, C. Raman, J.M. Vogels, W. Ketterle, Science 292, 5516 (2001).

[11] T.L. Ho, Phys. Rev. Lett. 87, 060403 (2001).

[12] E.J. Mueller, T.L. Ho, Phys. Rev. Lett. 88, 180403 (2002).

[13] J. Zhang, H. Zhai, Phys. Rev. Lett. 95, 200403 (2005).

[14] M.Ö. Oktel, Phys. Rev. A 69, 023618 (2004).

[15] H. Zhai, Q. Zhou, R. Lü, L. Chang, Phys. Rev. A 69, 063609 (2004).

[16] N.R. Cooper, E.H. Rezayi, S.H. Simon, Phys. Rev. Lett. 95, 200402 (2005).

[17] R. Barnett, S. Mukerjee, J.E. Moore, Phys. Rev. Lett. 100, 240405 (2008).

[18] P. Kuopanportti, J.A. M. Huhtamäki, M. Möttönen, Phys. Rev. A 85, 043613 (2012).

[19] M. Cipriani, M. Nitta, Phys. Rev. Lett. 111, 170401 (2013).

[20] Y.-J. Lin, R.L. Compton, A.R. Perry, W.D. Phillips, J.V. Porto, I.B. Spielman, Phys. Rev. Lett. 102 130401 (2009).

[21] Y.-J. Lin, R.L. Compton, K. Jiménez-García, J.V. Porto, I.B. Spielman, Nature 462, 628 (2009).

[22] R.M. Price, D. Trypogeorgos, D.L. Campbell, A. Putra, A. Valdés-Curiel, I.B. Spielman, New. J. Phys. 18, 113009 (2016).

[23] W. Bao, H.Q. Wang, P.A. Markowich, Commun. Math. Sci. 3, 57 (2005).

[24] A. Kato, Y. Nakano, K. Kasamatsu, T. Matsui, Phys. Rev. A 84, 053623 (2011).

[25] Q. Zhao, J. Low. Temp. Phys. 182, 117 (2016).

[26] S. Choi, S.A. Morgan, K. Burnett, Phys. Rev. A 57, 4057 (1998).

[27] M.-O. Mewes, M.R. Andrews, N.J. van Druten, D.M. Kurn, D.S. Durfee, C.G. Townsend, W. Ketterle, Phys. Rev. Lett. 77, 988 (1996).

[28] G. Thalhammer, G. Barontini, L. De Sarlo, J. Catani, F. Minardi, M. Inguscio, Phys. Rev. Lett. 100, 210402 (2008).

[29] C. Chin, R. Grimm, P. Julienne, E. Tiesinga, Rev. Mod. Phys. 82, 1225 (2010). 